\title{
Gerechter Frieden als Orientierungswissen - ein christliches Leitbild für eine plurale Gesellschaft? Ein Ausblick
}

Inhaltlich bewegt sich das Konzept des gerechten Friedens in dem entfalteten Spannungsfeld zwischen ziviler Konfliktbearbeitung und rechtserhaltender Gewalt. Welche Bedeutung kommt ihm nun aber als Orientierungswissen in innerund außerkirchlichen Kontexten zu? Die Friedensdenkschrift von 2007 gilt bis heute als zentrale Referenzquelle der EKD in Friedensfragen. Mit dem in ihr entwickelten Leitbild des gerechten Friedens beansprucht die Evangelische Kirche in Deutschland, der Gesellschaft ein Orientierungswissen in Friedensfragen bereitzustellen. So formulierte der damalige Ratsvorsitzender der EKD Wolfgang Huber in der Einleitung zur Friedensdenkschrift:

„In Denkschriften soll nach Möglichkeit ein auf christlicher Verantwortung beruhender, sorgfältig geprüfter und stellvertretend für die ganze Gesellschaft formulierter Konsens zum Ausdruck kommen“ (EKD 2007, S. 8).

Mit diesem Zitat verbinden sich zwei Annahmen beziehungsweise Ansprüche: 1) als Kirche ein Orientierungswissen für die gesamte Gesellschaft anzubieten und 2) als Kirche mit einer Stimme sprechen zu können. Beide Setzungen sind näher zu hinterfragen.

In einer immer komplexer werdenden Welt wird der Ruf nach Orientierung laut. Kirchliche Denkschriften sollen genau das bieten. Ihr erklärtes Ziel ist es, „Vorschläge und Angebote der Kirche für die öffentliche Diskussion“ (Huber 1973, S. 606) bereitzustellen, ,auf das öffentliche Bewußtsein in bestimmten Fragen Einfluß zu nehmen und so Voraussetzungen dafür zu schaffen, daß die zuständigen Organe handeln können“ (Huber 1973, S. 605). Nach dem Philosophen Andreas Luckner bedarf es dazu zweierlei: Reflexivität und Situativität. Ersteres beinhaltet eine vierfache Relation: „Jemand (1) orientiert sich (2) an etwas oder jemandem (3) in Bezug auf etwas (4)“(Luckner 2000, S. 66). Dabei sei 
Orientierung nur situativ möglich. Die ethische Grundfrage „Was soll ich tun?“ fokussiere sich im Orientierungswissen auf die Frage „Was ist zu tun in dieser Situation ratsam?“ (Luckners 2000, S. 69). Für die Urteilsbildung heißt dies, nicht allein auf eine ethische Normenreflexion zu setzen, sondern stets auch die empirischen Sachverhalte mit zu berücksichtigen und zueinander in Beziehung zu setzen. Mit Verweis auf Bruno Schüller (1980) spricht Thomas Hoppe (2021, i.E.) von „gemischten Normen“, ,,in denen sich ein moralisches Werturteil mit einem empirischen Tatsachenurteil verbindet". Vor diesem Hintergrund können Differenzen entweder auf Dissense moralischer Prinzipien oder aber auf strittige Einschätzungen hinsichtlich der empirischen Situation zurückgehen.

Auch die beiden dominierenden protestantischen sozialethischen Konzepte die Königsherrschaft Christi und die Zwei-Reiche-Lehre - stehen jeweils auf ihre Weise in der Gefahr, die „gemischten Normen“ nach der einen beziehungsweise anderen Seite aufzulösen. Während die Königsherrschaft Christi vor dem Problem steht, die Erkenntnisquellen ihrer Urteile allzu leicht als göttlichen Willen $\mathrm{zu}$ interpretieren und sich so den empirischen Tatsachen und dem Diskurs zu verschließen, tendiert die Zwei-Reiche-Lehre dazu, ,die Eigengesetzlichkeit der Weltverhältnisse“ zu überdehnen und sich den Möglichkeiten „eines begründeten Einspruchs gegen die in diesen Verhältnissen wirkenden Prozesslogiken“ zu berauben (Hoppe 2021, i.E.).

Welche der beiden sozialethischen Konzepte in Friedensfragen in Anschlag gebracht wird, ist stets auch eine ekklesiologische Frage nach der Sozialgestalt von Kirche. Und hier scheint sich in jüngster Zeit ein Wandel im Selbstverständnis zu vollziehen. Statt die spezifische Funktion der Kirche im Sinne eines öffentlichen Protestantismus darin zu sehen, ,den Boden für Kompromisse zu bereiten und den Sinn für die Suche nach dem Gemeinsamen zu pflegen“, scheint der Wunsch zu wachsen, ,eine bestimmte Position zu verstärken“ (Anselm 2021, i.E.).

Friedensfragen wurden auch in der Vergangenheit im kirchlichen Raum kontrovers debattiert: angefangen von der Wiederbewaffnung Deutschlands über die nukleare Abschreckung bis hin zum NATO-Doppelbeschluss 1979. Trotz dieser Kontroversen galt es jedoch, ,unter dem Evangelium zusammen [zu bleiben]“ (EKD-Synode 1958, zit. nach Härle 2011, S. 396). Vor diesem Hintergrund entstanden 1959 die Heidelberger Thesen. Die dort enthaltene Komplementaritätsthese, als Kompromissformel entwickelt, sollte die Kontroversen um die beiden einander ausschließenden Möglichkeiten - die Friedenssicherung durch militärische Mittel oder durch vollständigen Verzicht auf Gewalt - einhegen und das Entweder-Oder durch ein Sowohl-als-Auch ersetzen. 
Mit der Friedensdenkschrift von 2007 deutete sich bereits ein Wandel an. So ist mit der Absage an die Heidelberger Thesen auch der fast 50 Jahre währende friedensethische Kompromiss in Fragen nuklearer Abschreckung zugunsten eines Nuklearpazifismus aufgegeben worden (vgl. Werkner 2019). Einen weiteren Schritt in diese Richtung vollzog die EKD-Synode zum Frieden 2019. In ihrem Kundgebungstext konstatiert sie: „Das Leitbild des Gerechten Friedens setzt die Gewaltfreiheit an die erste Stelle“ (EKD 2019, S. 3). Was dieses Statement für das staatliche Gewaltmonopol und für christliche Soldatinnen und Soldaten der Bundeswehr bedeutet, bleibt unerwähnt; eine Verhältnisbestimmung wird nicht vorgenommen. Bernd Oberdorfer sieht darin ein Abrücken von der in der lutherischen Sozialethik gewichtigen Zwei-Reiche-Lehre hin zu einer ,Ethik der auf Gewaltfreiheit fokussierten Jesusnachfolge" (Oberdorfer 2021, i.E.) und einen Wandel ,,vom Deliberativen zum Prophetischen“ (Oberdorfer 2021, i.E.).

Und auch die „Zwölf Leitsätze zur Zukunft einer aufgeschlossenen Kirche“, beschlossen auf der EKD-Synode 2020, setzen auf einen Reformprozess, der Veränderungen im kirchlichen Selbstverständnis erkennen lässt. So will sich die evangelische Kirche nicht mehr einer „staatsanalogen Behörde“ ähneln, sondern sich zu einem ,innovationsorientierten Unternehmen“ beziehungsweise einer „handlungsstarken zivilgesellschaftlichen Organisation“ entwickeln (EKD 2020, S. 24). $\mathrm{Zu}$ gesellschaftlichen Prozessen wird sie öffentlich dort Stellung beziehen, wo dies - so die Einschränkung - ,,vom Evangelium her geboten ist und sich in unserem kirchlichen Leben und Handeln praktisch und erkennbar niederschlägt“ (EKD 2020, S. 11). Dabei will sich die EKD künftig ,um ein klares geistliches Profil in einer unübersichtlichen Welt“ bemühen: „Die Kirche wird umso glaubwürdiger, je mehr ihr Reden rückgebunden bleibt an ihr eigenes zeichenhaftes und richtungsweisendes Handeln“ (EKD 2020, S. 11). Ekklesiologisch können die „Zwölf Leitsätze“ im Sinne einer Abkehr von volkskirchlichen Strukturen gelesen werden. Hier zeichnet sich eine Entwicklung ab, ,Kirche nicht mehr als integrierendes, auf die Gesamtgesellschaft ausgerichtete Institution [zu verstehen]" (Anselm 2021, i.E.), sondern als eine soziale Bewegung oder NGO, die, von einer Mission getragen auf die Durchsetzung einer bestimmten Botschaft zielt. Die „Anwaltschaft für eine bestimmte Perspektive erlaubt es, eigene Positionen eindeutig und scharf $\mathrm{zu}$ formulieren und diese auch selbstbewusst in den politischen Diskurs einzuspielen“ (Anselm 2021, i.E.).

Noch ist offen, welche Entwicklung die Evangelische Kirche in Deutschland in den nächsten Jahren und Jahrzehnten nehmen wird. Der eben skizzierte und in Ansätzen auch schon eingeschlagene Weg hätte weitreichende Konsequenzen. 
Zwar könnten friedensethische und -politische Fragen in aller Klarheit und Eindeutigkeit in der Öffentlichkeit vertreten und in die Politik eingebracht werden, die Pluralität innerhalb der Kirche ginge damit aber verloren:

„Pluralismusfähig ist diese Organisationsform nur in der Weise, dass sie sich selbst dezidiert als eine spezifische Stimme im pluralen Konzert gesellschaftlicher Meinungsbildung versteht, nicht aber in dem Sinn, dass sie auch nach innen hin unterschiedliche Perspektiven abbilden wollte“ (Anselm 2021, i.E.).

Bei einer solchen Organisationsform wären zentrale staatskirchenrechtliche Privilegien kaum mehr zu rechtfertigen mit der Folge, dass es der Kirche weitaus schwerer fallen dürfte, sich in der Öffentlichkeit und im Konzert anderer zivilgesellschaftlichen Kräfte Gehör zu verschaffen. Dagegen könnte der Mehrwert einer Kirche als Volkskirche gerade darin liegen, in ihren friedensethischen Debatten „eine Abwägungskultur zu profilieren, die die gängigen dualistischen Streitmuster durchbricht, dabei aber nicht in eine normativ orientierungslose, vage EinerseitsAndererseits-Rhetorik abgleitet" (Ebeling 2021, i.E.). Dabei müsste noch sehr viel stärker der Prozesscharakter des Friedens und mit ihm die zeitliche Dimension in den Fokus der Analysen rücken.

Open Access Dieses Kapitel wird unter der Creative Commons Namensnennung 4.0 International Lizenz (http://creativecommons.org/licenses/by/4.0/deed.de) veröffentlicht, welche die Nutzung, Vervielfältigung, Bearbeitung, Verbreitung und Wiedergabe in jeglichem Medium und Format erlaubt, sofern Sie den/die ursprünglichen Autor(en) und die Quelle ordnungsgemäß nennen, einen Link zur Creative Commons Lizenz beifügen und angeben, ob Änderungen vorgenommen wurden.

Die in diesem Kapitel enthaltenen Bilder und sonstiges Drittmaterial unterliegen ebenfalls der genannten Creative Commons Lizenz, sofern sich aus der Abbildungslegende nichts anderes ergibt. Sofern das betreffende Material nicht unter der genannten Creative Commons Lizenz steht und die betreffende Handlung nicht nach gesetzlichen Vorschriften erlaubt ist, ist für die oben aufgeführten Weiterverwendungen des Materials die Einwilligung des jeweiligen Rechteinhabers einzuholen.

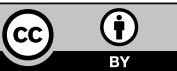

